\title{
Some weighted norm inequalities for a one-sided version of $g_{\lambda}^{*}$
}

by

L. DE Rosa and C. Segovia (Buenos Aires)

\begin{abstract}
We study the boundedness of the one-sided operator $g_{\lambda, \varphi}^{+}$between the weighted spaces $L^{p}\left(M^{-} w\right)$ and $L^{p}(w)$ for every weight $w$. If $\lambda=2 / p$ whenever $1<p<2$, and in the case $p=1$ for $\lambda>2$, we prove the weak type of $g_{\lambda, \varphi}^{+}$. For every $\lambda>1$ and $p=2$, or $\lambda>2 / p$ and $1<p<2$, the boundedness of this operator is obtained. For $p>2$ and $\lambda>1$, we obtain the boundedness of $g_{\lambda, \varphi}^{+}$from $L^{p}\left(\left(M^{-}\right)^{[p / 2]+1} w\right)$ to $L^{p}(w)$, where $\left(M^{-}\right)^{k}$ denotes the operator $M^{-}$iterated $k$ times.
\end{abstract}

1. Notations and definitions. As usual, $\mathcal{S}$ denotes the class of all those $C^{\infty}$-functions defined on $\mathbb{R}$ such that

$$
\sup _{x \in \mathbb{R}}\left|x^{m}\left(D^{n} \varphi\right)(x)\right|<\infty
$$

for all non-negative integers $m$ and $n$. We also consider the space $C_{0}^{\infty}$ of all $C^{\infty}$-functions defined on $\mathbb{R}$ with compact support.

If $E \subset \mathbb{R}$ is a Lebesgue measurable set, we denote its Lebesgue measure by $|E|$, and the characteristic function of $E$ by $\chi_{E}(x)$.

Let $f$ be a measurable function defined on $\mathbb{R}$. The one-sided HardyLittlewood maximal functions $M^{-} f$ and $M^{+} f$ are given by

$$
M^{-} f(x)=\sup _{h>0} \frac{1}{h} \int_{x-h}^{x}|f(t)| d t, \quad M^{+} f(x)=\sup _{h>0} \frac{1}{h} \int_{x}^{x+h}|f(t)| d t .
$$

A weight $w$ is a measurable and non-negative function defined on $\mathbb{R}$. If $E \subset \mathbb{R}$ is a measurable set, we denote its $w$-measure by $w(E)=\int_{E} w(t) d t$. Given $p \geq 1, L^{p}(w)$ is the space of all measurable functions $f$ such that

$$
\|f\|_{L^{p}(w)}=\left(\int_{-\infty}^{\infty}|f(x)|^{p} w(x) d x\right)^{1 / p}<\infty .
$$

If $w=1$, we simply write $L^{p}$ and $\|f\|_{L^{p}}$.

2000 Mathematics Subject Classification: Primary 42B25, 26A33.

Key words and phrases: one-sided maximal functions, Littlewood-Paley theory, onesided weights. 
We shall say that a function $B:[0, \infty) \rightarrow[0, \infty)$ is a Young function if it is continuous, convex, increasing and satisfies $\lim _{t \rightarrow \infty} B(t)=\infty$. The Luxemburg norm of a function $f$ is given by

$$
\|f\|_{B}=\inf \left\{\lambda>0: \int B(|f| / \lambda) \leq 1\right\}
$$

and the average over an interval $I$ is:

$$
\|f\|_{B, I}=\inf \left\{\lambda>0: \frac{1}{|I|} \int_{I} B(|f| / \lambda) \leq 1\right\} .
$$

The one-sided maximal operators associated to $B$ are defined as

$$
M_{B}^{+}(f)(x)=\sup _{h>0}\|f\|_{B,[x, x+h]}, \quad M_{B}^{-}(f)(x)=\sup _{h>0}\|f\|_{B,[x-h, x]} .
$$

Let $\varphi$ belong to $\mathcal{S}$ and be supported on $(-\infty, 0]$ with $\int \varphi(x) d x=0$. For every $\lambda>1$, the one-sided operator $g_{\lambda, \varphi}^{+}$was defined in [RoSe] as

$$
g_{\lambda, \varphi}^{+}(f)(x)=\left(\int_{0}^{\infty} \int_{x}^{\infty}\left(\frac{t}{t+y-x}\right)^{\lambda}\left|f * \varphi_{t}(y)\right|^{2} \frac{d y d t}{t^{2}}\right)^{1 / 2} .
$$

Throughout this paper the letter $C$ will always mean a positive constant not necessarily the same at each occurrence. If $1<p<\infty$ then $p^{\prime}$ denotes its conjugate exponent: $p+p^{\prime}=p p^{\prime}$.

2. Statement of the results. In [CW], S. Chanillo and R. Wheeden obtained the boundedness of the area integral between the spaces $L^{p}(M w)$ and $L^{p}(w)$ when $1<p \leq 2$. For $p=2$ and $\lambda>1$, if the support of $\varphi$ is compact, they showed in $\left[\mathrm{CW}\right.$, Lemma (1.1)] that the operator $g_{\lambda, \varphi}^{*}$ maps $L^{2}(M w)$ into $L^{2}(w)$. We shall give, in Theorem A, a one sided-version of this result without the restriction on the support of $\varphi$. For $1<p<2$ and $\lambda=2 / p$, in order to prove Theorem B below, we use some arguments due to C. Fefferman (see $[\mathrm{F}]$ ). As a consequence of Theorems A and $\mathrm{B}$, for $1<p \leq 2$ and $\lambda>2 / p$, we obtain, in Theorem $\mathrm{C}$, the boundedness of $g_{\lambda, \varphi}^{+}$between $L^{p}\left(M^{-} w\right)$ and $L^{p}(w)$. For $p>2$, the known techniques (see [P]) allow us to prove Theorem D.

Next, we state the already mentioned Theorems A-D.

Theorem A. Let $\varphi \in \mathcal{S}$ with $\operatorname{supp}(\varphi) \subset(-\infty, 0]$ and $\int \varphi(x) d x=0$. Then, for every $\lambda>1$,

$$
\left(\int_{-\infty}^{\infty} g_{\lambda, \varphi}^{+}(f)(x)^{2} w(x) d x\right)^{1 / 2} \leq C_{\lambda, \varphi}\left(\int_{-\infty}^{\infty}|f(x)|^{2} M^{-} w(x) d x\right)^{1 / 2}
$$

with a constant $C_{\lambda, \varphi}$ not depending on $f$.

Theorem B. Let $\varphi \in \mathcal{S}$ with $\operatorname{supp}(\varphi) \subset(-\infty, 0]$ and $\int \varphi(x) d x=0$. Let $\lambda>2$ if $p=1$, and $\lambda=2 / p$ whenever $1<p<2$. Then there exists a 
constant $C_{p, \lambda, w, \varphi}$ such that

$$
w\left(\left\{x: g_{\lambda, \varphi}^{+}(f)(x)>\mu\right\}\right) \leq \frac{C_{p, \lambda, w, \varphi}}{\mu^{p}} \int_{-\infty}^{\infty}|f(x)|^{p} M^{-} w(x) d x
$$

for every function $f$ and $\mu>0$.

Theorem C. Let $\varphi \in \mathcal{S}$ with $\operatorname{supp}(\varphi) \subset(-\infty, 0]$ and $\int \varphi(x) d x=0$. Let $1<p \leq 2$. If $\lambda>2 / p$, then there exists a constant $C_{p, \lambda, w, \varphi}$ such that

$$
\int_{-\infty}^{\infty} g_{\lambda, \varphi}^{+}(f)(x)^{p} w(x) d x \leq C_{p, \lambda, w, \varphi} \int_{-\infty}^{\infty}|f(x)|^{p} M^{-} w(x) d x
$$

for every function $f$.

Theorem D. Let $\varphi \in \mathcal{S}$ with $\operatorname{supp}(\varphi) \subset(-\infty, 0]$ and $\int \varphi(x) d x=0$. Let $\lambda>1$ and $p>2$. Then there exists a constant $C_{p, \lambda, w, \varphi}$ such that

$$
\int_{-\infty}^{\infty} g_{\lambda, \varphi}^{+}(f)(x)^{p} w(x) d x \leq C_{p, \lambda, w, \varphi} \int_{-\infty}^{\infty}|f(x)|^{p}\left(M^{-}\right)^{[p / 2]+1}(w)(x) d x .
$$

3. Proof of the results. The following lemma and remark will be used in the proof of Theorem A.

LEMmA 1. Let $\varphi \in C_{0}^{\infty}$ with $\operatorname{supp}(\varphi) \subset\left[-2^{s}, 0\right], s \geq 0$, and $\int \varphi(x) d x=0$. Then

$$
\int_{-\infty}^{\infty} g_{\lambda, \varphi}^{+}(f)(x)^{2} w(x) d x \leq C_{\lambda} 2^{s \lambda}\left(\int_{-\infty}^{\infty}|\widehat{\varphi}(t)|^{2} \frac{d t}{|t|}\right) \int_{-\infty}^{\infty}|f(x)|^{2} M^{-} w(x) d x
$$

with a constant $C_{\lambda}$ depending neither on $f$ nor on $\varphi$.

Proof. By Fubini's theorem, we have

$$
\begin{array}{rl}
\int_{-\infty}^{\infty} g_{\lambda, \varphi}^{+}(f)(x)^{2} & w(x) d x \\
& =\int_{-\infty}^{\infty} \int_{0}^{\infty} \int_{x}^{\infty}\left(\frac{t}{t+y-x}\right)^{\lambda}\left|f * \varphi_{t}(y)\right|^{2} \frac{d y d t}{t^{2}} w(x) d x \\
& =\int_{0}^{\infty} \int_{-\infty}^{\infty}\left|f * \varphi_{t}(y)\right|^{2}\left(\frac{1}{t} \int_{-\infty}^{y}\left(\frac{t}{t+y-x}\right)^{\lambda} w(x) d x\right) \frac{d y d t}{t} .
\end{array}
$$

For each integer $k$, we consider the set

$$
A_{k}=\left\{(y, t): 2^{k-1}<\frac{1}{t} \int_{-\infty}^{y}\left(\frac{t}{t+y-x}\right)^{\lambda} w(x) d x \leq 2^{k}\right\} .
$$


Then

$$
\int_{-\infty}^{\infty} g_{\lambda, \varphi}^{+}(f)(x)^{2} w(x) d x \leq \sum_{k \in \mathbb{Z}} 2^{k} \int_{0}^{\infty} \int_{-\infty}^{\infty}\left|f * \varphi_{t}(y)\right|^{2} \chi_{A_{k}}(y, t) \frac{d y d t}{t}
$$

For every $(y, t)$ belonging to $A_{k}$ and $y \leq z \leq y+2^{s} t$, we have

$$
\begin{aligned}
\frac{1}{t} \int_{-\infty}^{z}\left(\frac{t}{t+z-x}\right)^{\lambda} w(x) d x & \geq \frac{1}{2^{(s+1) \lambda}} \frac{1}{t} \int_{-\infty}^{y}\left(\frac{t}{t+y-x}\right)^{\lambda} w(x) d x \\
& >\frac{2^{k-1}}{2^{(s+1) \lambda}} .
\end{aligned}
$$

On the other hand, since $\lambda>1$, there exists a constant $C_{\lambda}$ such that for every $z$,

$$
\frac{1}{t} \int_{-\infty}^{z}\left(\frac{t}{t+z-x}\right)^{\lambda} w(x) d x \leq C_{\lambda} M^{-} w(z) .
$$

Therefore, if $(y, t) \in A_{k}$ and $y \leq z \leq y+2^{s} t$ then $z$ belongs to $E_{k}=\{z$ : $\left.M^{-} w(z) \geq\left(C_{\lambda} / 2^{(s+1) \lambda}\right) 2^{k-1}\right\}$. Taking into account that $\operatorname{supp}(\varphi) \subset\left[-2^{s}, 0\right]$, we get

$$
f * \varphi_{t}(y)=\int f(z) \chi_{E_{k}}(z) \varphi_{t}(y-z) d z=\left(f \chi_{E_{k}} * \varphi_{t}\right)(y) .
$$

Then, by Plancherel's and Fubini's theorems, (2) is majorized by

$$
\sum_{k \in \mathbb{Z}} 2^{k} \int_{0}^{\infty} \int_{-\infty}^{\infty}\left|f \chi_{E_{k}} * \varphi_{t}(y)\right|^{2} \frac{d y d t}{t}=\sum_{k \in \mathbb{Z}} 2^{k} \int_{-\infty}^{\infty}\left|\widehat{f \chi_{E_{k}}}(y)\right|^{2} \int_{0}^{\infty}|\widehat{\varphi}(t y)|^{2} \frac{d t}{t} d y
$$

The inner integral is bounded by $C_{\varphi}=\int_{-\infty}^{\infty}\left(|\widehat{\varphi}(t)|^{2} /|t|\right) d t$. Thus, applying Plancherel's theorem again, we get

$$
\int_{-\infty}^{\infty} g_{\lambda, \varphi}^{+}(f)(x)^{2} w(x) d x \leq C_{\varphi} \int_{-\infty}^{\infty}|f(y)|^{2} \sum_{k \in \mathbb{Z}} 2^{k} \chi_{E_{k}}(y) d y
$$

Finally, we observe that by the definition of $E_{k}$,

$$
\sum_{k \in \mathbb{Z}} 2^{k} \chi_{E_{k}}(y) \leq C_{\lambda} 2^{s \lambda} M^{-} w(y)
$$

for almost every $y$, ending the proof of the lemma.

REMARK. We observe that if $\varphi \in \mathcal{S}$ and $\int \varphi(x) d x=0$, then

$$
\int_{-\infty}^{\infty}|\widehat{\varphi}(s)|^{2} \frac{d s}{|s|} \leq 4 \pi^{2}\left(\int_{-\infty}^{\infty}|s||\varphi(s)| d s\right)^{2}+\int_{-\infty}^{\infty}|\varphi(s)|^{2} d s .
$$

In fact, since $\int \varphi(x) d x=0$, we have

$$
|\widehat{\varphi}(s)|=\left|\int_{-\infty}^{\infty} \varphi(t)\left(e^{-2 \pi i s t}-1\right) d t\right| \leq 2 \pi|s| \int_{-\infty}^{\infty}|t||\varphi(t)| d t .
$$


Consequently,

$$
\int_{|s| \leq 1}|\widehat{\varphi}(s)|^{2} \frac{d s}{|s|} \leq 4 \pi^{2}\left(\int_{-\infty}^{\infty}|s||\varphi(s)| d s\right)^{2} .
$$

On the other hand, in view of Plancherel's theorem

$$
\int_{|s| \geq 1}|\widehat{\varphi}(s)|^{2} \frac{d s}{|s|} \leq \int_{-\infty}^{\infty}|\widehat{\varphi}(s)|^{2} d s \leq \int_{-\infty}^{\infty}|\varphi(s)|^{2} d s
$$

which shows that (3) holds.

Let $\eta$ be a non-negative and $C_{0}^{\infty}$-function with support contained in $[-2,-1]$ and $\int \eta(x) d x=1$. For every non-negative integer $k$, let $\eta_{k}(x)=$ $2^{-k} \eta\left(2^{-k} x\right)$. We define

$$
\theta(x)=\int_{|x| / 2 \leq|t| \leq|x|} \eta(t) d t .
$$

Then $\theta \in C_{0}^{\infty}$ and $\operatorname{supp}(\theta) \subset[-4,-1] \cup[1,4]$. For every positive integer $k$, let

$$
\theta_{k}(x)=\theta\left(2^{-k+1} x\right)
$$

and for $k=0$, let

$$
\theta_{0}(x)=1-\int_{|y| \leq|x|} \eta(y) d y
$$

Then $\sum_{k=0}^{\infty} \theta_{k}(x)=1$ for every $x$. Given $\varphi \in \mathcal{S}$ with $\operatorname{supp}(\varphi) \subset(-\infty, 0]$ and $\int \varphi(x) d x=0$, we define

$$
a_{k}=\int \sum_{h=0}^{k} \theta_{h}(y) \varphi(y) d y, \quad k \geq 0, \quad a_{-1}=0 .
$$

For every non-negative integer $k$, let $\varrho_{k}$ be given by

$$
\varrho_{k}(x)=\theta_{k}(x) \varphi(x)+a_{k-1} \eta_{k-1}(x)-a_{k} \eta_{k}(x) .
$$

It is easy to check that $\operatorname{supp}\left(\varrho_{k}\right) \subset\left[-2^{k+1},-2^{k-1}\right]$ for $k \geq 1$, and $\operatorname{supp}\left(\varrho_{0}\right) \subset$ $[-2,0]$. Moreover, $\int \varrho_{k}(x) d x=0$ for every $k \geq 0$, and $\sum_{k=0}^{\infty} \varrho_{k}=\varphi$. We shall show that for every $N>2$,

$$
C_{\varrho_{k}}=\int_{-\infty}^{\infty}\left|\widehat{\varrho}_{k}(s)\right|^{2} \frac{d s}{|s|} \leq C_{N, \varphi} 2^{-2 k(N-2)} .
$$

By definition of $\varrho_{k}$,

$$
\begin{aligned}
\left(\int_{-\infty}^{\infty}\left|\varrho_{k}(x)\right|^{2} d x\right)^{1 / 2} & \leq\left(\int_{-\infty}^{\infty}\left|\theta_{k}(x) \varphi(x)\right|^{2} d x\right)^{1 / 2} \\
& +\left|a_{k-1}\right|\left(\int_{-\infty}^{\infty}\left|\eta_{k-1}(x)\right|^{2} d x\right)^{1 / 2}+\left|a_{k}\right|\left(\int_{-\infty}^{\infty}\left|\eta_{k}(x)\right|^{2} d x\right)^{1 / 2} .
\end{aligned}
$$


Since $0 \leq \theta_{k}(x) \leq 1$ and $\operatorname{supp}\left(\theta_{k} \varphi\right) \subset\left[-2^{k+1},-2^{k-1}\right]$ for $k \geq 1$, and $\operatorname{supp}\left(\theta_{0} \varphi\right) \subset[-2,0]$, we have

$$
\begin{aligned}
\left(\int_{-\infty}^{\infty}\left|\theta_{k}(x) \varphi(x)\right|^{2} d x\right)^{1 / 2} & \leq\left(\int_{\operatorname{supp}\left(\theta_{k} \varphi\right)} \frac{C_{N, \varphi}}{(1+|x|)^{2 N}} d x\right)^{1 / 2} \\
& \leq C_{N, \varphi} 2^{-k(N-1 / 2)}
\end{aligned}
$$

By definition of $a_{k}$, and taking into account that $\int \varphi(x) d x=0$, we get

$$
\begin{aligned}
\left|a_{k}\right| & =\left|-\int \sum_{h=k+1}^{\infty} \theta_{h}(y) \varphi(y) d y\right| \leq \int_{|y| \geq 2^{k}}|\varphi(y)| d y \\
& \leq C_{N, \varphi} \int_{|y| \geq 2^{k}} \frac{d y}{(1+|y|)^{N}} \leq C_{N, \varphi} 2^{-k(N-1)} .
\end{aligned}
$$

Thus,

$$
\left|a_{k}\right|\left(\int_{-\infty}^{\infty}\left|\eta_{k}(x)\right|^{2} d x\right)^{1 / 2}=\frac{\left|a_{k}\right|}{2^{k / 2}}\left(\int_{-\infty}^{\infty}|\eta(x)|^{2} d x\right)^{1 / 2} \leq C_{N, \varphi} 2^{-k(N-1 / 2)}
$$

Then, by (6)-(8),

$$
\int_{-\infty}^{\infty}\left|\varrho_{k}(x)\right|^{2} d x \leq C_{N, \varphi} 2^{-2 k(N-1 / 2)}
$$

Simple calculations show that

$$
\int_{-\infty}^{\infty}|x|\left|\varrho_{k}(x)\right|^{2} d x \leq C_{N, \varphi} 2^{-2 k(N-2)}
$$

Now, using (3) we obtain (5).

Proof of Theorem A. We consider the sequence of functions $\left\{\varrho_{k}, k \geq 0\right\}$ defined in (4). Since $\sum_{k=0}^{\infty} \varrho_{k}=\varphi$ and $\sum_{k=0}^{\infty} \chi_{\operatorname{supp}\left(\varrho_{k}\right)}(x) \leq 3$, we have

$$
f * \varphi_{t}(y)=\sum_{k=0}^{\infty} f *\left(\varrho_{k}\right)_{t}(y)
$$

for every $y$. Then

$$
\begin{aligned}
& \left(\int_{-\infty}^{\infty} g_{\lambda, \varphi}^{+}(f)(x)^{2} w(x) d x\right)^{1 / 2} \\
& \quad \leq \sum_{k=0}^{\infty}\left(\int_{-\infty}^{\infty} \int_{0}^{\infty} \int_{x}^{\infty}\left(\frac{t}{t+y-x}\right)^{\lambda}\left|f *\left(\varrho_{k}\right) t(y)\right|^{2} \frac{d y d t}{t^{2}} w(x) d x\right)^{1 / 2} \\
& \quad=\sum_{k=0}^{\infty}\left(\int_{-\infty}^{\infty} g_{\lambda, \varrho_{k}}^{+}(f)(x)^{2} w(x) d x\right)^{1 / 2}
\end{aligned}
$$


Keeping in mind that $\operatorname{supp}\left(\varrho_{k}\right) \subset\left[-2^{k+1}, 0\right]$ and $\int \varrho_{k}(x) d x=0$, we can apply Lemma 1 . Then, by the estimate (5) with $N>\lambda+2$, we find that (9) is bounded by a constant times

$$
\begin{aligned}
\sum_{k=0}^{\infty} 2^{(k+1) \lambda / 2}\left(\int_{-\infty}^{\infty}\left|\widehat{\varrho}_{k}(t)\right|^{2} \frac{d t}{|t|}\right)^{1 / 2} & \left(\int_{-\infty}^{\infty}|f(x)|^{2} M^{-} w(x) d x\right)^{1 / 2} \\
\leq & C_{\lambda, \varphi}\left(\int_{-\infty}^{\infty}|f(x)|^{2} M^{-} w(x) d x\right)^{1 / 2}
\end{aligned}
$$

In order to prove Theorem B, we shall need the following one-sided Fefferman-Stein type inequality and Lemma 11.

Lemma 10. There exists a positive constant $C$, such that

$$
w\left(\left\{x: M^{+}(f)(x)>\mu\right\}\right) \leq \frac{C}{\mu} \int_{-\infty}^{\infty}|f(x)| M^{-} w(x) d x
$$

for every function $f$, and $\mu>0$.

Proof. The proof is similar to the proof of Theorem 1 in [M, p. 693], and it shall not be given.

Lemma 11. Let $I=(\alpha, \beta)$, a bounded interval, $1<\lambda<2$, and $k \geq 4$. Then there exists a constant $C_{\lambda, k}$ such that for every $x<\alpha-2|I|$,

$$
\int_{0}^{\infty} \int_{x}^{\alpha-2|I|}\left(\frac{t}{t+y-x}\right)^{\lambda}\left(\frac{t}{t+\alpha-y}\right)^{k} \frac{d y d t}{t^{4}} \leq C_{\lambda, k} \frac{|I|^{\lambda-2}}{(\alpha-x)^{\lambda}}
$$

Proof. Changing the variables $(y, t)$ to

$$
z=(\alpha-y) / t \quad \text { and } \quad u=(\alpha-x) / t
$$

we obtain

$$
\begin{aligned}
& \int_{0}^{\infty} \int_{\alpha-x \geq \alpha-y \geq 2|I|}\left(\frac{1}{1+\frac{y-x}{t}}\right)^{\lambda}\left(\frac{1}{1+\frac{\alpha-y}{t}}\right)^{k} \frac{d y d t}{t^{4}} \\
& =\frac{1}{(\alpha-x)^{2}} \int_{0}^{\infty} \int_{u \geq z \geq 2|I| u /(\alpha-x)} \frac{1}{(1+u-z)^{\lambda}} \frac{1}{(1+z)^{k}} u d u d z
\end{aligned}
$$

We set $A=2|I| /(\alpha-x)$. Applying Fubini's theorem, it is enough to show that

$$
\int_{0}^{\infty} \frac{1}{(1+z)^{k}} \int_{z \leq u \leq z / A} \frac{u}{(1+u-z)^{\lambda}} d u d z \leq C_{\lambda, k} A^{\lambda-2} .
$$


Recalling that $1<\lambda<2$, we have

$$
\begin{aligned}
& \int_{0}^{\infty} \frac{1}{(1+z)^{k}} \int_{z \leq u \leq z / A, u-z>u / 2} \frac{u}{(1+u-z)^{\lambda}} d u d z \\
& \leq \int_{0}^{\infty} \frac{1}{(1+z)^{k}} \int_{0}^{z / A}\left(\frac{2}{u}\right)^{\lambda} u d u d z=C_{\lambda} \int_{0}^{\infty} \frac{1}{(1+z)^{k}}\left(\frac{z}{A}\right)^{2-\lambda} d z=A^{\lambda-2} .
\end{aligned}
$$

Since $k \geq 4, A<1$ and $\lambda<2$, it follows that

$$
\begin{aligned}
\int_{0}^{\infty} \frac{1}{(1+z)^{k}} & \int_{z \leq u \leq z / A, u-z \leq u / 2} \frac{u}{(1+u-z)^{\lambda}} d u d z \\
& \leq \int_{0}^{\infty} \frac{1}{(1+z)^{k}} \int_{0}^{2 z} u d u d z=2 \int_{0}^{\infty} \frac{z^{2}}{(1+z)^{k}} d z \leq C_{k} A^{\lambda-2}
\end{aligned}
$$

which ends the proof of the lemma.

Proof of Theorem B. By a density argument it is enough to consider $f \in$ $L^{p}\left(M^{-} w\right) \cap L^{p}$. It is well known that the set $\Omega=\left\{x: M^{+}\left(|f|^{p}\right)(x)^{1 / p}>\mu\right\}$ is open. Let $\left\{I_{j}\right\}_{j \geq 1}$ be its connected components. Since $f \in L^{p}$, each $I_{j}$ is a bounded interval, and it is well known (see [HSt, pp. 421-424]) that

$$
\frac{1}{\left|I_{j}\right|} \int_{I_{j}}|f(x)|^{p} d x=\mu^{p} .
$$

Given $I_{j}=\left(\alpha_{j}, \beta_{j}\right)$, we write $I_{j}^{-}=\left(\alpha_{j}-4\left|I_{j}\right|, \alpha_{j}\right)$. By (12), we have

$$
w\left(I_{j}^{-}\right)=\frac{1}{\mu^{p}} \int_{I_{j}}|f(x)|^{p} \frac{w\left(I_{j}^{-}\right)}{\left|I_{j}\right|} d x \leq \frac{5}{\mu^{p}} \int_{I_{j}}|f(x)|^{p} M^{-} w(x) d x .
$$

Therefore, if we define $\widetilde{\Omega}=\bigcup_{j \geq 1} I_{j} \cup I_{j}^{-}$, applying Lemma 10 we obtain

$$
\begin{aligned}
w(\widetilde{\Omega}) & \leq w(\Omega)+\sum_{j \geq 1} w\left(I_{j}^{-}\right) \\
& \leq \frac{C}{\mu^{p}} \int_{-\infty}^{\infty}|f(x)|^{p} M^{-} w(x) d x+\frac{5}{\mu^{p}} \sum_{j \geq 1} \int_{I_{j}}|f(x)|^{p} M^{-} w(x) d x \\
& \leq \frac{C}{\mu^{p}} \int_{-\infty}^{\infty}|f(x)|^{p} M^{-} w(x) d x .
\end{aligned}
$$

Consequently, it is enough to prove that

$$
w\left(\left\{x \notin \widetilde{\Omega}: g_{\lambda, \varphi}^{+}(f)(x)>\mu\right\}\right) \leq \frac{C}{\mu^{p}} \int_{-\infty}^{\infty}|f(x)|^{p} M^{-} w(x) d x .
$$


We define

$$
\begin{aligned}
g(x) & =f(x) \chi_{\Omega^{\mathrm{c}}}(x)+\sum_{j \geq 1}\left(\frac{1}{\left|I_{j}\right|} \int_{I_{j}} f\right) \chi_{I_{j}}(x), \\
b_{j}(x) & =\left(f(x)-\frac{1}{\left|I_{j}\right|} \int_{I_{j}} f\right) \chi_{I_{j}}(x), \quad j \geq 1 .
\end{aligned}
$$

Then $f=g+b$ where $b=\sum_{j \geq 1} b_{j}$.

By Chebyshev's inequality and applying Theorem A, we get

$$
\begin{aligned}
w\left(\left\{x \notin \widetilde{\Omega}: g_{\lambda, \varphi}^{+}(f)(x)>\right.\right. & \mu\}) \leq \frac{1}{\mu^{2}} \int_{\widetilde{\Omega}^{\mathrm{c}}} g_{\lambda, \varphi}^{+}(g)(x)^{2} w(x) d x \\
\leq & \frac{C}{\mu^{2}} \int_{-\infty}^{\infty}|g(x)|^{2} M^{-}\left(w \chi_{\widetilde{\Omega}^{\mathrm{c}}}\right)(x) d x \\
& =\frac{C}{\mu^{2}} \int_{-\infty}^{\infty}|g(x)|^{2-p}|g(x)|^{p} M^{-}\left(w \chi_{\widetilde{\Omega}^{\mathrm{c}}}\right)(x) d x
\end{aligned}
$$

We observe that $|g(x)| \leq \mu$ almost everywhere. Then, by the definition of $g$ and Hölder's inequality, (14) is bounded by

$$
\frac{C}{\mu^{p}}\left[\int_{\Omega^{\mathrm{c}}}|f(x)|^{p} M^{-}\left(w \chi_{\widetilde{\Omega}^{\mathrm{c}}}\right)(x) d x+\sum_{j \geq 1} \int_{I_{j}}\left(\frac{1}{\left|I_{j}\right|} \int_{I_{j}}|f(z)|^{p} d z\right) M^{-}\left(w \chi_{\widetilde{\Omega}^{\mathrm{c}}}\right)(x) d x\right] .
$$

It is easy to see that $M^{-}\left(w \chi_{\widetilde{\Omega}^{\mathrm{c}}}\right)(x) \leq C M^{-}(w)(z)$ for every $x, z \in I_{j}$. Thus,

$$
w\left(\left\{x \notin \widetilde{\Omega}: g_{\lambda, \varphi}^{+}(g)(x)>\mu\right\}\right) \leq \frac{C}{\mu^{p}} \int_{-\infty}^{\infty}|f(x)|^{p} M^{-} w(x) d x .
$$

We define $I_{j}^{*}=\left(\alpha_{j}-2\left|I_{j}\right|, \beta_{j}\right)$ for every $j \geq 1$. We can write

$$
g_{\lambda, \varphi}^{+}(b)(x) \leq g^{1}(x)+g^{2}(x),
$$

where

$$
\begin{aligned}
& g^{1}(x)=\left(\int_{0}^{\infty} \int_{x}^{\infty}\left(\frac{t}{t+y-x}\right)^{\lambda}\left|\sum_{i: y \notin I_{i}^{*}} b_{i} * \varphi_{t}(y)\right|^{2} \frac{d y d t}{t^{2}}\right)^{1 / 2} \\
& g^{2}(x)=\left(\int_{0}^{\infty} \int_{x}^{\infty}\left(\frac{t}{t+y-x}\right)^{\lambda}\left|\sum_{i: y \in I_{i}^{*}} b_{i} * \varphi_{t}(y)\right|^{2} \frac{d y d t}{t^{2}}\right)^{1 / 2} .
\end{aligned}
$$

Let us consider $g^{1}(x)$. Taking into account that $b_{i} * \varphi_{t}(y)=0$ if $y>\beta_{i}$, and $\int\left|b_{i}(z)\right| d z \leq 2\left|I_{i}\right| \mu$, it follows that

$$
\left|\sum_{i: y \notin I_{i}^{*}} b_{i} * \varphi_{t}(y)\right| \leq \frac{2 \mu}{t} \sum_{i: y \notin I_{i}^{*}, y<\beta_{i}}\left|I_{i}\right| \sup _{z \in I_{i}}\left|\varphi\left(\frac{y-z}{t}\right)\right| .
$$


Since $\varphi \in \mathcal{S}$, and $\operatorname{supp}(\varphi) \subset(-\infty, 0]$, we deduce that

$$
\left|\varphi\left(\frac{y-z}{t}\right)\right| \leq \frac{C}{\left(1+\frac{w-y}{t}\right)^{2}} \quad \text { for } y \notin I_{i}^{*} \text { and } z, w \in I_{i} .
$$

Then

$$
\left|\sum_{i: y \notin I_{i}^{*}} b_{i} * \varphi_{t}(y)\right| \leq \frac{C \mu}{t} \sum_{i: y \notin I_{i}^{*}, y<\beta_{i} I_{i}} \int_{\frac{d w}{\left(1+\frac{w-y}{t}\right)^{2}} \leq c \mu .}
$$

Therefore,

$$
g^{1}(x)^{2} \leq C \mu \int_{0}^{\infty} \int_{x}^{\infty}\left(\frac{t}{t+y-x}\right)^{\lambda}\left|\sum_{i: y \notin I_{i}^{*}} b_{i} * \varphi_{t}(y)\right| \frac{d y d t}{t^{2}}=C \mu F(x),
$$

and by Chebyshev's inequality we get

$$
w\left(\left\{x \notin \widetilde{\Omega}: g^{1}(x)>\mu\right\}\right) \leq \frac{C}{\mu} \int_{\widetilde{\Omega}^{c}} F(x) w(x) d x .
$$

Since $\int b_{i}(z) d z=0$, applying the mean value theorem, for every $y \leq \alpha_{i}-2\left|I_{i}\right|$ we obtain the estimate

$$
\begin{aligned}
\left|b_{i} * \varphi_{t}(y)\right| & \leq \frac{1}{t} \int\left|b_{i}(z)\right|\left|\varphi\left(\frac{y-z}{t}\right)-\varphi\left(\frac{y-\alpha_{i}}{t}\right)\right| d z \\
& \leq \frac{C}{t} \int_{I_{i}}\left|b_{i}(z)\right|\left|\frac{z-\alpha_{i}}{t}\right|\left(\frac{t}{t+\alpha_{i}-y}\right)^{4} d z \\
& \leq C\left|I_{i}\right| \frac{t^{2}}{\left(t+\alpha_{i}-y\right)^{4}} \int_{I_{i}}|f(z)| d z .
\end{aligned}
$$

Then, by the definition of $F(x),(17)$ is majorized by

$$
\begin{aligned}
\frac{C}{\mu} \sum_{i \geq 1} \int_{I_{i}}|f(z)| d z \int_{\widetilde{\Omega}^{\mathrm{c}}}\left|I_{i}\right| \int_{0}^{\infty} \int_{x<y<\beta_{i}, y \notin I_{i}^{*}} & \left(\frac{t}{t+y-x}\right)^{\lambda^{\prime}} \\
& \times \frac{1}{\left(t+\alpha_{i}-y\right)^{4}} d y d t w(x) d x
\end{aligned}
$$

where $1<\lambda^{\prime}<\inf (\lambda, 2)$. Now, applying Lemma 11 with $k=4$, we find that (18) is bounded by

$$
\frac{C}{\mu} \sum_{i \geq 1} \int_{I_{i}}|f(z)| d z \int_{-\infty}^{\alpha_{i}-4\left|I_{i}\right|} \frac{\left|I_{i}\right|^{\lambda^{\prime}-1}}{\left(\alpha_{i}-x\right)^{\lambda^{\prime}}} w(x) \chi_{\widetilde{\Omega}^{\mathrm{c}}}(x) d x .
$$

The inner integral is bounded by $C M^{-}\left(w \chi_{\widetilde{\Omega}^{c}}\right)\left(\alpha_{i}\right)$. It is easy to verify that, by Hölder's inequality and (12), 


$$
\frac{1}{\mu} \int_{I_{i}}|f| \leq \frac{1}{\mu^{p}} \int_{I_{i}}|f|^{p}
$$

Thus, we obtain

$$
\begin{aligned}
w\left(\left\{x \notin \widetilde{\Omega}: g^{1}(x)>\mu\right\}\right) & \leq \frac{C}{\mu^{p}} \sum_{i} \int_{I_{i}}|f(z)|^{p} d z M^{-}\left(w \chi_{\widetilde{\Omega}^{\mathrm{c}}}\right)\left(\alpha_{i}\right) \\
& \leq \frac{C}{\mu^{p}} \int_{-\infty}^{\infty}|f(z)|^{p} M^{-} w(z) d z .
\end{aligned}
$$

Now, let us consider $g^{2}(x)$. By (12), there exists an integer $k_{0}$ such that $\left|I_{j}\right| \leq\|f\|_{p}^{p} \mu^{-p} \leq 2^{k_{0}}$ for every $j \geq 1$. Let $A_{k}=\left\{j: 2^{k-1}<\left|I_{j}\right| \leq 2^{k}\right\}$, $k \leq k_{0}$. We can write

$$
\bigcup_{j \geq 1} I_{j}^{*}=\bigcup_{k \leq k_{0}} \bigcup_{j \in A_{k}} E_{j}^{*}
$$

where $E_{j}^{*}=I_{j}^{*} \backslash \bigcup_{l>k} \bigcup_{s \in A_{l}} I_{s}^{*}$ for each $j \in A_{k}$. We observe that if $I_{i}^{*} \cap E_{j}^{*}$ is not empty then $I_{i}^{*} \subset I_{j}^{\prime}$, where $I_{j}^{\prime}$ is the interval with the same center of $I_{j}$ and with measure $20\left|I_{j}\right|$. For each $x \notin \widetilde{\Omega}$, we have

$$
g^{2}(x)^{2}=\sum_{k \leq k_{0}} \sum_{j \in A_{k}} \int_{0}^{\infty} \int_{x<y, y \in E_{j}^{*}}\left(\frac{t}{t+y-x}\right)^{\lambda}\left|\sum_{i: y \in I_{i}^{*}} b_{i} * \varphi_{t}(y)\right|^{2} \frac{d y d t}{t^{2}} .
$$

We observe that if $x \notin \widetilde{\Omega}^{\mathrm{c}}, x<y$ and $y \in E_{j}^{*}$ then $x<\alpha_{j}-4\left|I_{j}\right|$ and $t+y-x \geq\left(\alpha_{j}-x\right)-\left(\alpha_{j}-y\right) \geq\left(\alpha_{j}-x\right) / 2$. Then

$$
\begin{aligned}
g^{2}(x)^{2} \leq & C \sum_{k \leq k_{0}} \sum_{j \in A_{k}, x<\alpha_{j}} \frac{1}{\left(\alpha_{j}-x\right)^{\lambda}} \\
& \times \int_{0}^{\infty} \int_{x<y, y \in E_{j}^{*}} t^{\lambda-2}\left|\sum_{i: y \in I_{i}^{*}} b_{i} * \varphi_{t}(y)\right|^{2} d y d t .
\end{aligned}
$$

If we define $D_{j}=\bigcup_{i: E_{j}^{*} \cap I_{i}^{*} \neq \emptyset} I_{i}$ and $b^{j}(x)=|b(x)| \chi_{D_{j}}(x)$ then, for every $y \in E_{j}^{*}$, we obtain

$$
\begin{aligned}
\left|\sum_{i: y \in I_{i}^{*}} b_{i} * \varphi_{t}(y)\right| & \leq \sum_{i: y \in I_{i}^{*} I_{i}} \int|b(z)|\left|\varphi_{t}(y-z)\right| d z \\
& \leq \int_{\bigcup_{i: E_{j}^{*} \cap I_{i}^{*} \neq \emptyset} I_{i}}|b(z)|\left|\varphi_{t}(y-z)\right| d z \\
& \leq \int_{D_{j}}|b(z)|\left|\varphi_{t}(y-z)\right| d z=\left(b^{j} *|\varphi|_{t}\right)(y) .
\end{aligned}
$$


Consequently, by (20), we have

$$
\begin{aligned}
g^{2}(x)^{2} \leq & C \sum_{k \leq k_{0}} \sum_{j \in A_{k}, x<\alpha_{j}} \frac{1}{\left(\alpha_{j}-x\right)^{\lambda}} \\
& \times \int_{0}^{\infty} \int_{x<y, y \in E_{j}^{*}} t^{\lambda-2}\left|\left(b^{j} *|\varphi|_{t}\right)(y)\right|^{2} d y d t .
\end{aligned}
$$

We claim that

$$
\int_{0}^{\infty} \int_{E_{j}^{*}} t^{\lambda-2}\left|\left(b^{j} *|\varphi| t\right)(y)\right|^{2} d y d t \leq C\left|E_{j}^{*}\right|^{\lambda-2 / p}\left\|b^{j}\right\|_{p}^{2} .
$$

In fact, by Fubini's theorem, we have

$$
\begin{aligned}
\int_{0}^{\infty} t^{\lambda-2} \mid\left(b^{j}\right. & *|\varphi| t)\left.(y)\right|^{2} d t \\
& =\int_{y}^{\infty} b^{j}(z) \int_{y}^{\infty} b^{j}(w) \int_{0}^{\infty} t^{\lambda-4}|\varphi|\left(\frac{y-z}{t}\right)|\varphi|\left(\frac{y-w}{t}\right) d t d w d z .
\end{aligned}
$$

Since $\varphi \in \mathcal{S}$, and $\lambda<3$,

$$
\begin{aligned}
\int_{0}^{\infty} t^{\lambda-4}|\varphi|\left(\frac{y-z}{t}\right)|\varphi| & \left(\frac{y-w}{t}\right) d t \\
& \leq C \int_{0}^{\infty} t^{\lambda-4} \frac{1}{\left(1+\frac{z-y}{t}\right)^{2}} \frac{1}{\left(1+\frac{w-y}{t}\right)^{2}} d t \\
& \leq C \int_{0}^{\infty} \frac{t^{\lambda-4}}{\left(1+\frac{z+w-2 y}{t}\right)^{2}} d t=C_{\lambda}(z+w-2 y)^{\lambda-3}
\end{aligned}
$$

Then the left hand side of (22) is bounded by

$$
\begin{aligned}
& C \int_{E_{j}^{*}} \int_{y}^{\infty} b^{j}(z) \int_{y}^{\infty} b^{j}(w) \frac{1}{(z+w-2 y)^{3-\lambda}} d w d z d y \\
& \leq C^{\prime} \int_{E_{j}^{*}}^{\infty} \int_{y}^{\infty} \frac{b^{j}(z)}{(z-y)^{(3-\lambda) / 2}} d z \int_{y}^{\infty} \frac{b^{j}(w)}{(w-y)^{(3-\lambda) / 2}} d w d y \\
& \leq C^{\prime} \int_{E_{j}^{*}}\left|I_{(\lambda-1) / 2}^{+}\left(b^{j}\right)(y)\right|^{2} d y
\end{aligned}
$$

where $I_{(\lambda-1) / 2}^{+}$denotes the one-sided fractional integral operator of order $(\lambda-1) / 2$. In the case $1<p<2$ and $\lambda=2 / p$, since, as is well known, $I_{(\lambda-1) / 2}^{+}$is a bounded operator from $L^{p}$ to $L^{2}$, it follows that $(22)$ holds. 
For $2<\lambda<3$, the operator $I_{(\lambda-1) / 2}^{+}$maps $L^{1}$ into weak- $L^{2 /(3-\lambda)}$. Then, by Kolmogorov's condition (see [GRu, p. 485]), we obtain (22).

On the other hand, since $\int\left|b_{i}(y)\right|^{p} d y \leq(2 \mu)^{p}\left|I_{i}\right|$, we have

$$
\left\|b^{j}\right\|_{p} \leq\left(\sum_{i: E_{j}^{*} \cap I_{i}^{*} \neq \emptyset}(2 \mu)^{p}\left|I_{i}\right|\right)^{1 / p} \leq 2 \mu\left|I_{j}^{\prime}\right|^{1 / p}=C \mu\left|I_{j}\right|^{1 / p} .
$$

Therefore, by (21) and (22) we get

$$
g^{2}(x)^{2} \leq C^{\prime} \mu^{2} \sum_{k \leq k_{0}} \sum_{j \in A_{k}, x<\alpha_{j}} \frac{\left|I_{j}\right|^{\lambda}}{\left(\alpha_{j}-x\right)^{\lambda}} .
$$

Consequently,

$$
\begin{aligned}
w\left(\left\{x \notin \widetilde{\Omega}: g^{2}(x)>\mu\right\}\right) & \leq C \sum_{j}\left|I_{j}\right|^{\lambda} \int_{-\infty}^{\alpha_{j}-4\left|I_{j}\right|} \frac{w(x) \chi_{\widetilde{\Omega}^{\mathrm{c}}}(x)}{\left(\alpha_{j}-x\right)^{\lambda}} d x \\
& \leq \frac{C}{\mu^{p}} \sum_{j} \int_{I_{j}}|f(z)|^{p} d z M^{-}\left(w \chi_{\widetilde{\Omega}^{\mathrm{c}}}\right)\left(\alpha_{j}\right) \\
& \leq \frac{C}{\mu^{p}} \int_{-\infty}^{\infty}|f(z)|^{p} M^{-} w(z) d z .
\end{aligned}
$$

From (15), (16), (19) and (23) we deduce that (13) holds for $\lambda=2 / p$ if $1<p<2$ and for $2<\lambda<3$ if $p=1$. Taking into account that if $\lambda_{1} \leq \lambda_{2}$ then $g_{\lambda_{2}, \varphi}^{+}(f)(x) \leq g_{\lambda_{1}, \varphi}^{+}(f)(x)$, the proof of the theorem is complete.

We now deduce Theorem $\mathrm{C}$ from Theorems A and B.

Proof of Theorem $C$. The case $p=2$ and $\lambda>1$ was considered in Theorem A. Let $1<p<2$ and $2 / p<\lambda<2$. We have $\lambda=2 / q$ with $1<q<p$. Then, by Theorem $\mathrm{B}, g_{\lambda, \varphi}^{+}$maps $L^{q}\left(M^{-} w\right)$ into weak- $L^{q}(w)$. Since $g_{\lambda, \varphi}^{+}$is bounded from $L^{2}\left(M^{-} w\right)$ to $L^{2}(w)$, by interpolation, we get the assertion for $\lambda<2$. The case $\lambda \geq 2$ follows by simple arguments.

The following remark shows that for $\lambda=2$ and $p=1$, a weak type inequality as in Theorem B cannot be valid.

Remark. Let $\varphi \neq 0$ belong to $\mathcal{S}$ with $\operatorname{supp}(\varphi) \subset[-1,0]$ and $\int \varphi(x) d x$ $=0$. There exists $f \in L^{1}$ such that $g_{2, \varphi}^{+}(f)(x)=\infty$ for every $x$ belonging to an unbounded set.

In fact, we consider

$$
f(t)=\left(\frac{1}{|t| \ln ^{3 / 2}(1 /|t|)}-c\right) \chi_{[-1 / 2,0]}(t)
$$


where $c$ is the unique constant such that $\int f(t) d t=0$. For every $x<-4$, we have

$$
g_{2, \varphi}^{+}(f)(x)^{2} \geq \frac{1}{(1-x)^{2}} \int_{0}^{1} \int_{-2}^{0}\left|f * \varphi_{t}(y)\right|^{2} d y d t
$$

The support of $f * \varphi_{t}$ is contained in $(-\infty, 0]$ and the fractional integral $I_{1 / 2}(f) \notin L^{2}$ (see [Z, p. 232]). Then Plancherel's theorem yields

$$
\begin{aligned}
A:=\int_{0}^{\infty} \int_{-\infty}^{0}\left|f * \varphi_{t}(y)\right|^{2} d y d t & =\int_{0}^{\infty} \int_{-\infty}^{\infty}|\widehat{\varphi}(t y)|^{2}|\widehat{f}(y)|^{2} d y d t \\
& \geq C_{\varphi} \int_{-\infty}^{\infty} \frac{|\widehat{f}(y)|^{2}}{|y|} d y \\
& =C_{\varphi} \int_{-\infty}^{\infty}\left|I_{1 / 2}(f)(y)\right|^{2} d y=\infty
\end{aligned}
$$

Applying the mean value theorem, for every $y \leq-2$ we obtain

$$
\begin{aligned}
\left|f * \varphi_{t}(y)\right| & \leq \frac{1}{t} \int_{-1 / 2}^{0}|f(z)|\left|\varphi\left(\frac{y-z}{t}\right)-\varphi\left(\frac{y}{t}\right)\right| d z \\
& \leq \frac{1}{t} \int_{-1 / 2}^{0}|f(z)| \frac{|z|}{t} C_{\varphi}\left(\frac{t}{t+|y|}\right)^{2} d z \leq C \frac{1}{(t+|y|)^{2}} .
\end{aligned}
$$

Using these inequalities we get

$$
A_{1}:=\int_{0}^{\infty} \int_{-\infty}^{-2}\left|f * \varphi_{t}(y)\right|^{2} d y d t \leq C \int_{0}^{\infty} \int_{-\infty}^{-2} \frac{1}{(t+|y|)^{4}} d y d t<\infty .
$$

Since $\left|f * \varphi_{t}(y)\right| \leq \frac{1}{t}\|\varphi\|_{\infty}\|f\|_{1}$, we have

$$
A_{2}:=\int_{1}^{\infty} \int_{-2}^{0}\left|f * \varphi_{t}(y)\right|^{2} d y d t \leq C \int_{1}^{\infty} \int_{-2}^{0} \frac{1}{t^{2}} d y d t<\infty .
$$

By (24) and the estimates obtained for $A, A_{1}$, and $A_{2}$ it follows that $g_{2, \varphi}^{+}(f)(x)=\infty$ for every $x<-4$.

To prove Theorem D, we proceed as in Theorem 1.10 of [P, p. 150].

Proof of Theorem D. More generally, we shall prove that

$$
\int_{-\infty}^{\infty} g_{\lambda, \varphi}^{+}(f)(x)^{p} w(x) d x \leq C \int_{-\infty}^{\infty}|f(x)|^{p} M_{B}^{-}\left(w^{2 / p}\right)(x)^{p / 2} d x
$$


where $B$ is a Young function that satisfies

$$
\int_{c}^{\infty}\left(\frac{t^{p / 2}}{B(t)}\right)^{(p / 2)^{\prime}-1} \frac{d t}{t}<\infty
$$

In the case $B(t) \approx t^{p / 2}\left(1+\ln ^{+} t\right)^{[p / 2]}$, we get Theorem $\mathrm{D}$.

Let $r=p / 2$. We have

$$
I=\left\|g_{\lambda, \varphi}^{+}(f)\right\|_{L^{p}(w)}^{2}=\left\|g_{\lambda, \varphi}^{+}(f)^{2} w^{1 / r}\right\|_{L^{r}}=\int_{-\infty}^{\infty} g_{\lambda, \varphi}^{+}(f)(x)^{2} w(x)^{1 / r} g(x) d x,
$$

for some $g \in L^{r^{\prime}}$ with unit norm. We recall that

$$
M^{-}\left(g_{1} g_{2}\right)(x) \leq M_{B}^{-}\left(g_{1}\right)(x) M_{\bar{B}}^{-}\left(g_{2}\right)(x),
$$

where $\bar{B}$ is the complementary function to $B$. Then Theorem A and Hölder's inequality yield

$$
\begin{aligned}
I & \leq C \int_{-\infty}^{\infty}|f(x)|^{2} M^{-}\left(w^{1 / r} g\right)(x) d x \\
& \leq C \int_{-\infty}^{\infty}|f(x)|^{2} M_{B}^{-}\left(w^{1 / r}\right)(x) M_{\bar{B}}^{-}(g)(x) d x \\
& \leq C\left(\int_{-\infty}^{\infty}|f(x)|^{p} M_{B}^{-}\left(w^{1 / r}\right)(x)^{p / 2} d x\right)^{2 / p}\left(\int_{-\infty}^{\infty} M_{\bar{B}}^{-}(g)(x)^{r^{\prime}} d x\right)^{1 / r^{\prime}} \\
& =C\|f\|_{L^{p}(v)}^{2}\left\|M_{\bar{B}}^{-}(g)\right\|_{L^{r^{\prime}}},
\end{aligned}
$$

where $v=M_{B}^{-}\left(w^{1 / r}\right)(x)^{r}$. By Theorem 2.6 in [RiRoT], if $B$ satisfies $(25)$, then

$$
I \leq C\|f\|_{L^{p}(v)}^{2}\|g\|_{L^{r^{\prime}}} \leq C\|f\|_{L^{p}(v)}^{2} .
$$

It is easy to check that $M_{B}^{-}\left(w^{1 / r}\right)(x)^{r}=M_{\widetilde{B}}^{-}(w)(x)$, where $\widetilde{B}(t)=B\left(t^{1 / r}\right)$. If $\widetilde{B}(t)=t\left(1+\ln ^{+} t\right)^{[r]}$ then $B$ satisfies (25), and by Proposition 2.15 in [RiRoT] there exist two constants $C_{1}$ and $C_{2}$ such that

$$
C_{1} M_{\widetilde{B}}^{-}(w)(x) \leq\left(M^{-}\right)^{[r]+1} w(x) \leq C_{2} M_{\widetilde{B}}^{-}(w)(x),
$$

which completes the proof.

\section{References}

[CW] S. Chanillo and R. Wheeden, Some weighted norm inequalities for the area integral, Indiana Univ. Math. J. 36 (1987), 277-294.

[F] C. L. Fefferman, Inequalities for strongly singular convolution operators, Acta Math. 124 (1970), 9-36. 
[GRu] J. García Cuerva and J. L. Rubio de Francia, Weighted Norm Inequalities and Related Topics, North-Holland, Amsterdam 1985.

[HSt] E. Hewitt and K. Stromberg, Real and Abstract Analysis, Springer, New York, 1965.

[M] F. J. Martín-Reyes, New proofs of weighted inequalities for the one-sided HardyLittlewood maximal functions, Proc. Amer. Math. Soc. 117 (1993), 691-698.

[P] C. Pérez, Banach function spaces and the two-weight problem for maximal functions, in: Function Spaces, Differential Operators and Nonlinear Analysis (Paseky, 1995), Prometheus, Praha, 1996, 141-158.

[RiRoT] M. S. Riveros, L. de Rosa and A. de la Torre, Sufficient conditions for one-sided operators, J. Fourier Anal. Appl. 6 (2000), 607-621.

[RoSe] L. de Rosa and C. Segovia, One-sided Littlewood-Paley theory, ibid. 3 (1997), 933-957.

[Z] A. Zygmund, Trigonometrical Series, Monografje Mat., Warszawa-Lwów, 1935.

Juan José Olleros 29698 B

1426 Buenos Aires, Argentina

E-mail: liliana_de_rosa@yahoo.com.ar
Departamento de Matemática Facultad de Ciencias Exactas y Naturales Universidad de Buenos Aires 1428 Buenos Aires, Argentina and

Instituto Argentino de Matemática

CONICET

Saavedra 15 3er Piso

1083 Buenos Aires, Argentina

E-mail: segovia@iamba.edu.ar

Received August 14, 2000

Revised version February 14, 2006 